\title{
Non-Cardiogenic Pulmonary Edema
}

\author{
J. Gonzales and A. Verin \\ Georgia Health Sciences University, Augusta, Georgia \\ United States
}

\section{Introduction}

Pulmonary edema (PE) remains one of the more common reasons for admission to the hospital. Pulmonary edema is either cardiac or non-cardiac. The cardiac causes of pulmonary edema occur because the cardiac pump function has failed and there is increased capillary hydrostatic pressure secondary to elevated pulmonary venous pressure. Cardiogenic pulmonary edema is the accumulation of fluid with a low-protein content in the lung interstitium and alveoli and occurs when the pulmonary venous and left atrial venous return exceeds left ventricular output. Most often this is due to left heart failure, cardiac valve disease, volume overload, kidney failure or cardiac tamponade. Non cardiogenic pulmonary edema (NCPE) is a condition that is associated with high morbidity and mortality. Pulmonary edema fluid accumulates in the lungs through damaged capillary endothelial cells and this leads to impaired gas exchange (oxygen and carbon dioxide) with hypoxia and respiratory failure. The best example of non-cardiogenic pulmonary edema is acute respiratory distress syndrome (ARDS) (Sartori et al, 2010). ARDS is a serious condition of hypoxia, bilateral lung infiltrates on chest roentgenogram with subsequent respiratory failure. The hallmark of ALI (acute lung injury)/ARDS on the cellular level is pulmonary capillary endothelial cell permeability and fluid leakage into the pulmonary parenchyma, followed by neutrophils, cytokines and an acute inflammatory response. It is associated with a high morbidity and 30-50\% mortality. ARDS has multiple causes with the most common being sepsis or pneumonia, less common causes of ARDS with ensuing pulmonary edema are trauma or pancreatitis (Ware \& Mathay, 2005). Other causes of NCPE in hospitalized patients are intravenous fluid with volume overload, neurogenic pulmonary edema, reperfusion pulmonary edema, re-expansion pulmonary edema, opiate overdose, salicylate toxicity. Less common, forms of NCPE are high altitude pulmonary edema (HAPE), immersion pulmonary edema and negative pressure pulmonary edema (NPPE).

\section{Increased permeability pulmonary edema}

NCPE causes direct injury to the lungs in several forms. Under normal conditions fluid outflow that occurs from the lung capillaries through tiny gaps in the vascular endothelial cell (EC) junction is removed from the interstitial space and returned to the circulation by the lymphatic system. Physiologically, the main forces regulating fluid balance in the lungs are the microvascular pressure of the capillaries. Fluid leaves the capillaries and enters the pulmonary interstitium in proportion to the net capillary hydrostatic pressure minus the net 
osmotic pressure across the vessel wall. The formula for filtration across the pulmonary capillary semi-permeable membrane is

$$
\mathrm{Q}=\mathrm{K}-[\mathrm{CPmv}-\mathrm{Ppmv})-(\Pi m v-\Pi p m v)]
$$

which is different from the systemic capillary fluid exchange. $Q=$ net transvascular flow of fluid, K=membrane permeability, Pmv=hydrostatic pressure in the microvessels, Ppmv=hydrostatic pressure in the peri-microvascular interstitium, $\Pi m v=$ plasma protein osmotic pressure in the circulation, $\Pi p m v=$ protein osmotic pressure in the perimicrovascular interstitium. This equation only reflects the known hydrostatic pressures without including the lymph hydrostatic pressure which has been studied but is unknown in relation to the Starling forces equation (Sartori et al, 2010). It is known that one of the main functions of the lymphatic system is to return plasma proteins from the interstitial tissue space to the bloodstream. Analyses of blood plasma and lymph have shown that all of the proteins that are found in the plasma are also found in the lymph although in lower concentrations (Ono et al, 2005). In normal circumstances, low pulmonary capillary hydrostatic pressure provides a "safety" factor against pulmonary edema across the lung tissue.

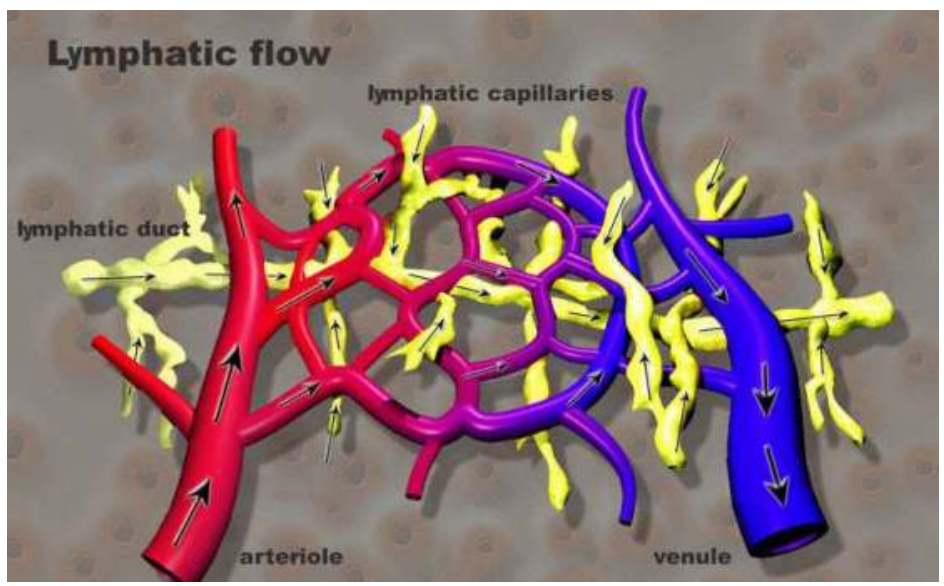

Fig. 1. Representation of the pulmonary arterial and venous capillary barrier and lymphatic flow.

Although hydrostatic pressures in the pulmonary arterial and venous system are known, the hydrostatic pressures of the interrelated pulmonary lymphatic system is unknown and largely unstudied. The illustration generously permitted by Dr. Davide Brunelli of Medars.it.

In some forms of NCPE such as ARDS disturbances of pulmonary capillary fluid balance and pulmonary permeability occur as a direct result of endotoxins and inflammation that cause disruption in the capillary EC barrier with barrier disruption and subsequent pulmonary venous congestion. As the volume excess initially enters the interstitium it is taken up by the lymphatic system to be returned to the vascular system. In normal circumstances the interstitial space can increase its volume by as much as $40 \%$ without resulting in pulmonary edema (Sartori et al, 2010). In pulmonary injury due to toxins or 
inflammation the fluid volume increase overwhelms the lymphatic drainage system and the hydrostatic forces become altered resulting in further injury to the pulmonary capillary endothelium. The result is persistent fluid accumulation that overwhelms the lymphatic drainage and tissue edema results. The final step occurs as the fluid volume overwhelms the hydrostatic forces and the excess fluid flows into the alveoli. The edema that is caused by the increasing fluid and vascular permeability is a hallmark of inflammation and tissue injury (Zimmerman \& McIntyre, 2004). The edema formation can have severe consequences because the fluid and protein components in the edematous tissues and alveoli increase the diffusion barrier for oxygen and carbon dioxide with subsequent disruption of gas exchange thus precipitating hypoxia and respiratory failure. The rate of volume expansion is also a factor in pulmonary edema.

Other forms of NCPE such as HAPE, immersion pulmonary edema and NPPE may occur due to greatly altered thoracic pressures and are thought to have a largely hydrostatic noninflammatory component (Fremont et al, 2007). The accelerated pulmonary edema that occurs with HAPE, immersion PE or NPPE is thought to be due to significant fluid shifts that come secondary to changes in intrathoracic pressure, and in the case of HAPE or immersion PE, possibly due to diminished atmospheric or oxygen inhalation pressures. Physiologically negative intrathoracic pressure is generated in the chest when a patient inspires against an obstructed airway. The rapid pressure change in the pulmonary venous circulation alters the transpulmonary fluid gradient. The increase in capillary hydrostatic pressure most likely causes capillary gap formation as well as transcellular fluid shifts. The fluid gradient is thus going from a high gradient to a low gradient. Although considered a second mechanism of the EC barrier disruption it is part of the capillary hydrostatic pressure. While it is unlikely that the rapidly resolved post obstructive PE and HAPE are of a different nature, it is more likely that all of the non-cardiogenic PE have the same hydrostatic mechanisms initially (Sartori et al, 2010 \& Gantor et al, 2006). The NCPE in ARDS is ultimately a result of capillary permeability secondary to cellular damage, inflammatory cascades, and over inflation by mechanical ventilation resulting in endothelial permeability. The permeability from HAPE and scuba diving is not initiated by inflammation but rather stress failure occurring due to the increased pressures that occur at the capillary level in healthy subjects (Slade et al, 2001). The pulmonary capillary endothelial cell barrier is a semi-permeable membrane and is known to be an active biological interface between the blood and the surrounding tissue. The EC is a single layer of continuous endothelium lining the pulmonary capillaries and forms a single layer between blood and the pulmonary interstitium. The pulmonary capillaries have extremely thin walls to allow rapid exchange of respiratory gases across them (Costello et al, 1992).The endothelium modulates tone, growth, homeostasis and inflammation in the lungs and throughout the circulatory system (Ware \& Matthay, 2005 \& Umapathy et al, 2010). In NCPE due to diseases such as ARDS, the ensuing endotoxins and inflammatory markers induce capillary endothelial disarray and gap formations. This is followed by neutrophil chemotaxis, diapedesis and protein rich edema fluid leakage into the interstitial spaces. Inflammatory cascades are triggered and also enter into the interstitial milieu. As the capillary pressure increases there is damage of the capillary EC causing larger molecules such as proteins from the vascular space to flow into the interstitial space (Slade et al, 2001). NCPE in an ill patient with ARDS is a high protein pulmonary edema. The fluid/plasma ratio may be used to differentiate the etiology of pulmonary edema in NCPE and CPE. It is a measurement of the alveolar fluid, obtained by broncho- alveolar lavage (BAL), to the serum plasma during 
acute pulmonary edema and has been shown in studies to be sensitive enough to differentiate a low protein fluid that results from CPE or NCPE in a patient without illness compared to a high protein ratio NCPE such as occurs with illness such as endotoxin induced ARDS (Fremont et al, 2001; Ware et al, 2010). The protein concentration in the pulmonary interstitium of ARDS exceeds $60 \%$ of the plasma value whereas the protein concentration in HAPE, reperfusion PE, neurogenic PE and other non ARDS causes is less than $45 \%$ (Fein et al, 1079 \& Staub et al, 1967). The result is capillary injury with gap formation and high permeability of the EC barrier with an increase of protein rich edema fluid into the interstitial space. The resulting pulmonary interstitial protein remains elevated compared to circulating blood plasma. At this point there is no longer a "quick" resolution possible.

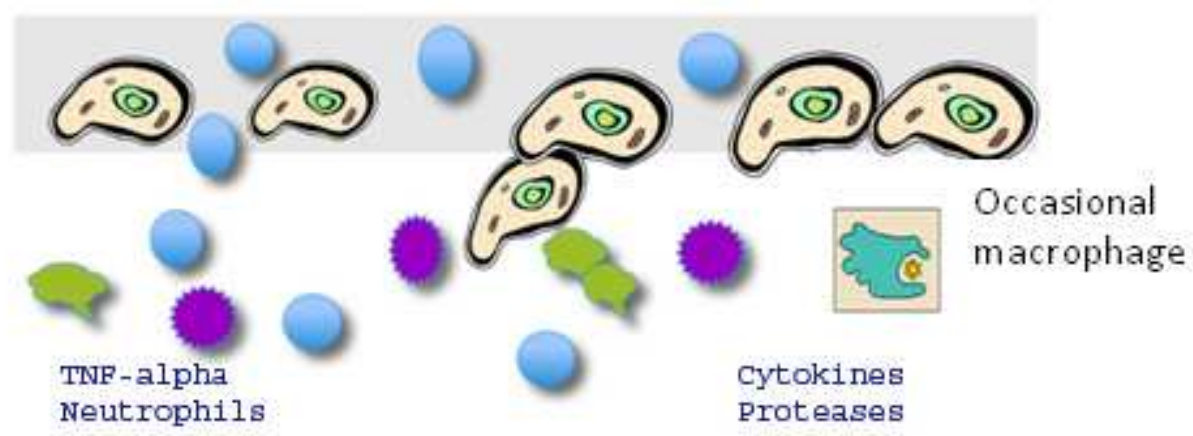

Fig. 2. Schematic representation of the endothelial barrier in inflammatory pulmonary edema.

NCPE causes direct injury to the lungs. The endothelial barrier is normally single layer of continuous endothelium lining the pulmonary capillaries. In ALI/ARDS the endotoxins recruited by the macrophages and neutrophils induce capillary endothelial disarray and gap formations. This is followed by neutrophil chemotaxis, diapedesis, proteases, cytokines and protein rich fluid into the interstitial spaces.

\section{Neutrophils and molecular mechanisms in the endothelial and epithelial cell barrier in ALI/ARDS}

Neutrophils play an important role in the development of pulmonary edema associated with ALI/ARDS. It is well known that neutrophils are prevalent in ARDS pulmonary edema and are central to the pathogenesis (Bdeir et al, 2010). Initially pulmonary macrophages are activated and recruit circulating neutrophils to the pulmonary microvascular system and the injured capillaries. The interaction of the neutrophils and the endothelium is initiated by adhesion receptors and by soluble or membrane bound chemoattractants. Intercellular adhesion molecules-1 (ICAM-1) and other proteins are known to be present but the specific role of these molecules and proteins is unclear (Downey et al, 1999). Once the neutrophils become activated and move subsequently into the pulmonary parenchymal interstitium, they sequester and initiate an important component of the inflammatory response in endotoxin induced ALI/ARDS. This activation 
and migration of neutrophils is a characteristic event in the progression of ALI and ARDS. Animal studies have shown that endothelial injury appears within minutes to hours after ALI initiation with resulting intercellular gaps of the EC. The EC gaps allow for permeability of fluid, neutrophils and cytokines into the pulmonary parenchymal space (Grommes \& Soehnlein, 2011).

The neutrophils that infiltrate the lungs and migrate into the airways express proinflammatory cytokines such as TNF- $\alpha$, IL-1 $\beta$, IL- 6 and contribute to both the endothelial and epithelial integrity disruption of the barriers (Bdeir et al, 2010; Grommes \& Soehnlein, 2011; Abraham, 2003). It has also been well documented that the percentage of neutrophils correlates directly with the alveolar-arterial PO2 difference in ALI/ARDS pulmonary edema (Weiland et al, 1986). Neutrophil sequestration is aided by chemotactic factors and by the adhesion molecules on both the neutrophils and capillary endothelial cells (Hasko et al, 2006; Steinberg, 1994; Geerts et al, 2001). The activated neutrophils expressing IL-1 $\beta$ produce other pro-inflammatory cytokines after endotoxin administration. In fact, the removal of neutrophils after endotoxin administration almost entirely prevents an increase of IL-1 $\beta$ expression and attenuates endotoxin induced TNF-a. Neutrophils are the major source of IL-1 $\beta$ in murine models of the lung in ALI (Abraham, 2003). Another feature of the neutrophils that accumulate in the lung of murine models is increased activation of the transcriptional regulatory factor NF-KB. NF-KB is a protein complex that controls transcription of DNA and is involved in cellular responses to stimuli such as pulmonary edema due to ALI/ARDS. It is key in regulating the endotoxin induced immune response in neutrophils and produces increased amounts of pro-inflammatory cytokines whose transcription is dependent on NF-KB (Blackwell et al, 1996). Neutrophils also become an increasing liability in the edematous pulmonary interstitium as they release free radicals.

The alveolar-capillary barrier is a very thin membrane allowing oxygen $\mathrm{CO}_{2}$ exchange for normal respiration. The major consequence of pulmonary edema is impaired gas exchange that interrupts the normal fluid exchange balance. The alveoli epithelium removes fluid by molecular mechanisms of sodium transport, however, the capillary endothelial barrier function has only incompletely defined pathways affecting the concurrent barrier disruption. Permeability of the EC in the capillaries with concurrent alveolar-capillary membrane damage and with leakage of fluid, neutrophils, proteases, cytokines and free radicals that all contribute to the ensuing pulmonary edema is a prominent feature of permeability edema and ALI/ARDS (Holter et al, 1986). The alveolar liquid clearance from the alveolus into the interstitium is based on active sodium transport largely through the highly regulated apical amiloride sensitive epithelial sodium channel complex (ENaC) with concomitant passive water transport and the $\mathrm{Na}^{+}, \mathrm{K}^{+}$ATPase exchange (Elia et al, 2003; Folkesson \& Matthay, 2006). The $\mathrm{Na}^{+}, \mathrm{K}^{+}$ATPase exchange transports the alveolar liquid into the interstitium and ultimately into the lymphatic and blood vessels (Hamacher et al, 2010; Lucas et al, 2009). However, these transport processes are often impaired in ALI or ARDS.

It is likely that the induction of increased permeability of the pulmonary capillary bed is directly linked to reversible physical modifications of the pulmonary capillary endothelium (Kaner et al, 2000). The capillary endothelial regulation of endothelial permeability involves various pathways such as those involving reactive oxygen species (ROS), Rho GTPases, and tyrosine phosphorylation of junctional proteins all converge to regulate junctional permeability. They either affect the stability of junctional proteins or modulate their interactions (Lucas et al, 2009). The regulation of permeability at the junctions is mediated 
by active communication between the proteins of the adherens junctions and the actin cytoskeleton. Actin mediated endothelial cell contraction is the result of myosin light chain (MLC) phosphorylation by MLC kinase (MLCK) in $\mathrm{Ca}^{2+} /$ calmodulin- dependent manner. RhoA also potentiates MLC phosphorylation by inhibiting MLC phosphatase activity through its downstream effector Rho kinase (ROCK). As the actin/myosin driven contraction generates a contractile force it pulls VE-cadherin inward. This contraction will force VE-cadherin to dissociate from its adjacent partner causing endothelial gaps - the basic pathology in permeability pulmonary edema (Lucas et al, 2009).

\section{Serum Biomarkers in permeability edema and ARDS}

A complex progression of events is recognized in the development of permeability edema and ARDS but the exact nature of events is still an area of active study. A large variety of inflammatory mediators have been found to be elevated in ARDS including lung specific proteins, endotoxin binding proteins, tumor necrosis factor alpha (TNF- $\mathrm{a}$ ), interleukins (ILs), chemokines and markers of endothelium activation such as adhesion molecules and von Willebrand factor antigen (VWF) (Tzouvelekis et al, 2005). A comprehensive review is beyond the scope of this article but some of the most widely known are discussed. There is an increased expression of the vascular endothelial growth factor VEGF gene in pulmonary edema. Although VEGF is widely expressed in the body, the highest level of expression in normal tissues is in the lung. Normally increased expression and angiogenesis is associated with lung tumors and has been studied as a target for therapy in lung cancer however VEGF also is known to stimulate actin stress fiber formation and new focal adhesions in endothelial cells suggesting a regulatory role in endothelial morphology (Kaner et al, 2000). A study by Kaner et al demonstrated that excess expression of VEGF within the murine lung was associated with increased permeability of pulmonary edema (Kaner et al, 2000). In addition to endotoxin effects on endothelial permeability there is strong evidence that cytokines such as tumor necrosis factor (TNF- $\alpha$ ), interleukin (IL)-1 $\beta$, IL-6, and IL-8 are associated with pulmonary edema. Shutte measured cytokine levels in patients with ARDS, severe pneumonia and cardiogenic pulmonary edema for comparison and found consistently higher levels of IL-8, IL-6 and TNF- $\alpha$ in the bronchoalveolar lavage fluid (BALF) and the serum of patients with ARDS and pneumonia compared to cardiogenic pulmonary edema (Schutte et al, 1996). Cytokines have various effects on activating endothelium inducing endothelial expression of adhesion molecules and leukocyte chemotaxis leading to a local inflammatory response in the lung. The cytokine, TNF- $\alpha$ induces macrophages and $\mathrm{T}_{\mathrm{H}} 1$ cells and activates ECs and macrophages (Braun et al, 2005). Studies have been conflicting as far as elevation of TNF- $\alpha$ in patients with ARDS pulmonary edema, for example, Bauer demonstrated that TNF- $\alpha$ concentrations were significantly higher in patients with ARDS than those of pneumonia or of the control subjects (Bauer et al, 2000). Others, such as Hyers have found variability in the elevation of TNF- $\alpha$, however, there is speculation that TNF- $\alpha$ is an acute phase cytokine and the timing of studies is important for its evaluation (Hyers, 1991). Although Bauer found that the TNF-a levels were significantly elevated it was also speculated that the elevation was more related to the severity of the lung disease and could not be extrapolated as a possible diagnostic marker for ARDS (Bauer et al, 2000). TNF-a is a known acute phase reactant and is also a cytokine involved in systemic inflammation. It is able to induce apoptotic cell death, inflammation, and inhibit tumorgenesis and viral replication. TNF- $\alpha$ is produced mainly by macrophages 
but can also be produced by other cells including endothelial cells (Schutte et al, 1996). Large amounts of TNF-a are released in response to LPS endotoxin, bacterial products and IL-1. TNF- a works with IL-1 and IL-6 to produce actions on various organ systems (Schutte et al, 1996; Braun et al, 2005; Bauer et al, 2000). TNF-a also induces EC activation and barrier dysfunction both of which occur in the pathogenesis of pulmonary edema and ALI/ARDS. It can promote edema by TNF receptor dependent chemokine production and adhesion molecule expression and leads to neutrophil chemotaxis (Braun et al, 2005; Ward, 1996). It also causes a decrease in transendothelial electrical resistance across human pulmonary artery EC (HPAEC) (Petrache et al 2003). The pulmonary capillary EC have a balanced system between contracting and tethering forces that normally act to protect the EC barrier from paracellular gaps. The balancing forces depend on cytoskeletal components such as actin based microfilaments, intermediate filaments and microtubules. TNF- $\alpha$ causes contraction via the actin filaments and this results in the formation of gaps and EC permeability (Braun 2005). In pulmonary edema associated with ARDS, TNF- $a$ can mediate acute inflammation and edema formation. It can also have a beneficial effect by increasing alveolar fluid clearance via an amiloride sensitive, cAMP independent mechanism to enhance alveolar fluid clearance. This is accomplished by binding to its receptors or activating $\mathrm{Na}+$ channels in the epithelium (Fukuda et al, 2001).

Other networks of cytokines regulate lung inflammation in lung injury and edema. Complement, $\mathrm{C} 5 \mathrm{a}$ and or the membrane attack complex, C5b-9 can directly activate EC to up-regulate adhesion molecules (P-selectin) or act synergistically with TNF-a to up regulate I-CAM and E Selectin (Shutte et al, 1996 \& Ward, 1996). IL-4 and IL-10 suppress TNF-a and can have a strong attenuating effect on TNF-a. Studies have shown that blocking IL-10 increased TNF-a production, neutrophil recruitment and the intensity of lung inflammation (Ward, 1996). Although the role of cytokines in the pathogenesis of ARDS has been widely recognized, their importance in the clinical diagnosis has not been clearly defined.

Recent studies have identified that the receptor for advanced glycation end products (RAGE) is activated by its ligands in many disorders including ALI/ARDS. RAGE and interaction with the high mobility group box-1 (HMGB-1)- one of its ligands- promotes local lung endothelial inflammation and evokes both local and systemic inflammation (Nakamura et al, 2011 \& Wolfson et al, 2010). In vitro studies determined that RAGE is the primary receptor signaling HMGB-1 induced endothelial barrier disruption and endothelial gap formation in human pulmonary artery endothelial cells (Wolfson et al, 2010). Soluable RAGE (sRAGE) has also been noted in the plasma of patients with ARDS and it was investigated as a biomarker of severity and clinical outcomes in patients with ARDS. In addition sRAGE and HMGB-1 levels were elevated in non- survivors compared to survivors in ARDS (Nakamura et al, 2011). An analysis of biomarker levels in two randomized controlled trials of ventilator therapy for ALI was done and the biomarkers that were elevated were ICAM-1, von Willebrand factor, IL-8, soluble TNF receptor-1, and surfactant protein-D. It was concluded that combining three or more biomarkers may be useful for selecting a high risk ALI group of patients (Calfee et al, 2011). Biomarker identification of risk remains an area of intense research.

\section{Non-inflammatory NCPE}

Categories of NCPE that resolve more quickly than ARDS and NCPE due to infection, trauma or other medical illness are high altitude pulmonary edema (HAPE), Immersion 
pulmonary edema (SCUBA diving and swimming) and negative pressure pulmonary edema (NPPE). Most commonly the non-inflammatory forms of NCPE occur in baseline healthy people although there may be a predilection to development of pulmonary edema in some people. However, there is no known way to predict who will develop pulmonary edema and who will not in the same circumstances.

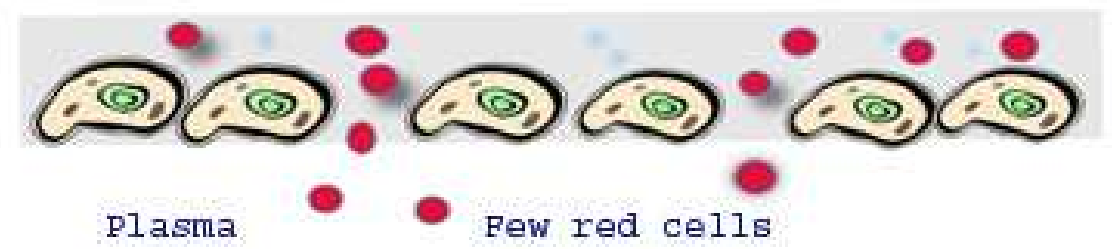

Fig. 3. Schematic representation of the endothelial barrier in Non-inflammatory Pulmonary Edema

Non- inflammatory pulmonary edema may have an element of hemorrhage leading to a pink frothy tint but will not initially have an inflammatory secretion of cells. The pulmonary edema resolves quickly and the endothelial barrier is thought to accommodate hydrostatic changes that resolve quickly compared to toxin mediated changes that heal slowly and may develop fibrinous changes.

\section{HAPE}

HAPE is a life threatening form of NCPE that has a rapid onset in healthy people who venture to elevations above 8200 feet. This occurs when the lower barometric pressures result in hypoxia, usually less than $90 \% \mathrm{SPO}_{2}$ or $60 \mathrm{PaO}_{2}$. Studies done at high altitude on patients who developed HAPE and compared to those who did not, have shown that pulmonary artery pressures are elevated and lead to a protein rich and mildly hemorrhagic edema. Leukocytes, cytokines, nitric oxide metabolites and eicosanoids are normal when compared to control subjects who did not develop HAPE (Swenson et al, 2002). The mechanism of the increased pulmonary vasoconstriction and resulting increased pulmonary pressures is not resolved. The mechanism is thought to be increased pulmonary and arterial capillary pressures second to hypoxic pulmonary vasoconstriction. Another effect is a noninflammatory increase in the permeability of the vascular endothelium. Some people are more susceptible to HAPE than others but the differentiating factors are not known. The most important treatment is to descend as soon as possible and provide oxygen therapy. Other treatments are Dexamethasone and nifedipine. Phosphodiesterase inhibitors are effective but the side effects worsen mountain sickness headaches (Maggiorini, 2010).

\section{Immersion PE}

Immersion PE that occurs in diving with self contained underwater breathing apparatus (SCUBA), and some triathlon athletes, combat swimmers (such as found in military missions) and breath hold divers is another type of non-inflammatory NCPE. Only about 1$2 \%$ of immersion divers develop PE and studies have not shown clearly what makes some people susceptible. One study did show that women, hypertension, fish oil and asthma may 
be factors that predispose to this condition (Miller III et al, 2010). The mechanism is poorly understood but reviews of cases have shown that the onset is rapid and that rapid improvement is also seen, similar to patients who develop HAPE (Slade et al, 2001). This observation lends credence to the probability that this is an acute but transient increase in pressure. The stress from the increased transalveolar pressure gradient that occurs with immersion is such that the non-cardiogenic capillary endothelium layer cell develops leaks (Slade et al, 2001). It has been shown that even asymptomatic dives result in an increased accumulation of extravascular lung water (Marinovic et al, 2010). They further demonstrated an increase in lung water, pulmonary artery pressure, NT-proBNP levels and decreased left ventricular contractility in healthy study volunteers who did not develop pulmonary edema. Immersion is known to increase preload and cold exposure increases both preload and afterload by vasoconstriction; it is possible that the changes could lead to damage in the pulmonary endothelium and lead to intrapulmonary redistribution of blood with regional overperfusion of some pulmonary capillaries and stress failure increasing capillary damage and permeability (Marinovic et al, 2010 \& Pons et al, 1995). Hyperoxia and low tank pressures are other possible mechanisms that may damage the pulmonary endothelium and increase endothelial permeability the development of pulmonary edema in immersion PE.

\section{Negative pressure pulmonary edema}

Negative pressure pulmonary edema (NPPE) is pulmonary edema that occurs following an acute upper airway obstruction and may also be referred to as a post obstructive pulmonary edema. It often occurs in otherwise healthy patients. The most common cause is laryngospasm soon after extubation from an endo-tracheal intubation in about $0.1 \%$ in post anesthesia patients (Pathak et al, 2011). There are also case reports of NPPE after other causes such as foreign body, epiglottitis, tracheal secretions, upper airway tumors, obesity, obstructive sleep apnea, tumors (Fremont et al, 2007). Following an episode of obstruction of the airway there is a marked increase in negative intrathoracic pressure against the obstructed upper airway. The rapid increase in intrathoracic pressure causes a rise in venous return to the right heart, which increases pulmonary venous volume and pulmonary venous pressure which then increases the pulmonary capillary transmural pressure. Systemic pressures also rise due to catecholamine induced veno-constriction from anxiety, hypoxia and hypercarbia (Schwartz et al, 1999). Fremont et al have shown that the mechanism is due to hydrostatic changes with the fluid moving from high pressure in the pulmonary venous system to low pressure into the pulmonary interstitium and airspaces. Studies confirmed that the pulmonary edema fluid to plasma protein ratio were consistent with hydrostatic causes of acute fluid shifts and pulmonary edema. This causes an increase of blood flow (venous return) to the right heart increasing pulmonary venous pressure (Fremont et al, 2007). The treatment is resolving the obstruction, oxygen and either reintubation or if the patient is stable CPAP may be used. The issue is not volume overload and diuretics are not indicated (Kapoor, 2011).

\section{Clinical manifestation of non cardiogenic pulmonary edema}

Clinically non-cardiogenic pulmonary edema is a permeability edema. The Starling equation predicts that a change in permeability of the microvascular membrane will result in an 
increase in the amount of fluid and protein that leaves the vascular space and enters the interstitial space. When the interstitial fluid increases in the interstitium then the outward movement continues and fluid enters the alveolar spaces through the tight junctions of the epithelium. Many entities are associated with permeability edema manifested as ARDS, the most common entities are sepsis, pneumonia, multiple blood transfusions, gastric aspiration, trauma, drug overdose and pancreatitis. and others. Clinically NCPE prototype ALI/ARDS presents with progressive hypoxia and respiratory failure. Multi-organ failure is frequent and one of the reasons the mortality is so high. ARDS affects 200,000 people per year and has a mortality rate of $30-50 \%$. This was initially described in the 1960 s' $^{\prime}$ by Petty and Ashbaugh (Ashbaugh et al, 1967). The diagnosis is made by four clinical criteria, acute onset of bilateral chest infiltrates, hypoxia, no evidence of left atrial hypertension (or clinical manifestations indicating left heart failure) and a ratio of arterial oxygen to fraction of inspired oxygen (PaO2/FIO2 ratio) of 201-300 for ALI and less than 200 for ARDS (Bernard et al, 1994 \& The Acute Respiratory Distress Syndrome Network, 2000). Due to the profound respiratory failure most patients require mechanical ventilation and care in the intensive care unit (ICU). The best therapeutic approach for ARDS permeability edema is to find and treat the cause. There is no pharmacological treatment for ARDS, the only "treatment" that has emerged since it was first described in 1971 is the ARDS-net trial protocol of low tidal volume mechanical ventilation. This is a strategy that is called "lung-protective ventilation". The main benefit of lung-protective ventilation is to avoid further injury to the lungs from high tidal volumes often used for patients in the ICU as these large tidal volumes increase the injury and subsequently cause an increase in the permeability pulmonary edema in the injured lung. The standard tidal volumes used in ICU have been $10-15 \mathrm{mg} / \mathrm{kg}$ for all mechanically ventilated patients, however, low tidal volumes of $6-8 \mathrm{mg} / \mathrm{kg}$ are recommended for patients with ARDS. A mortality benefit was one of the primary outcomes demonstrated in the ARDS-net trial for patients with ARDS who are mechanically ventilated at these low tidal volumes (Matthay et al, 2002 \& Marino 2007). Another aspect of NCPE and ARDS is heterogeneity of the lung parenchyma and the alveoli. The chest roentgenogram shows bilateral infiltrates that are difficult to distinguish from cardiogenic pulmonary edema and sometimes from pneumonia. The computed tomographic (CT) images are not necessarily diagnostic but in a patient with known ARDS shows that the lung edema and consolidation is not homogeneous but involves various lung regions, while other regions appear normal (Rouby et al, 2003). Alveolar over-distension from mechanical ventilation as well as repeated opening and collapse of the alveoli has been shown to cause lung injury initiating an increase in capillary stress and an increase in pro-inflammatory cytokine cascades (Matthay et al, 2002 \& Slutsky \&Tremblay, 1998). Hemodynamic evaluation of $\mathrm{NCPE}$ and ARDS requires careful ongoing evaluation, and may require monitoring cardiac filling pressures and cardiac output using a pulmonary artery catheter or currently, a noninvasive evaluation is more likely to be used. Diuretic therapy is then tailored to achieve the lowest cardiac filling pressures that do not compromise cardiac output and systemic oxygen transport. The nature of the lung interstitial infiltration is an inflammatory process, so that diuretic therapy to remove fluid does not remove the inflammation. Fluid treatment versus diuresis is not necessarily the goal of treatment in NCPE secondary to ARDS (Marino, 2007). Ultimately the treatment is supportive care using the appropriate ventilator strategies, promoting oxygenation, and treating the multi organ failure that is often contributes to the mortality. Histologically in this acute phase of lung injury there is widespread interstitial and alveolar edema with an abundance of neutrophils, erythrocytes, macrophages, cell 
debris, plasma proteins and strands of fibrin. At this phase there is injury to the capillary endothelium and denuding of the alveolar epithelium. If the patient survives this phase the pulmonary edema may completely resolve within a few months. Other patients with ALI/ARDS progress to a subacute phase over one to two weeks and may continue to have respiratory failure with hypoxia requiring mechanical ventilation. These patients develop fibrosis and capillary obliteration in the lungs, a condition called fibrosing alveolitis. These patients continue to progress with respiratory failure although they may recover from the initial event.

Progress from bench to bedside is being made; the mortality from NCPE and ALI/ARDS has improved from the initial $60 \%$ or more to $30-50 \%$. The establishment of the NIH ARDS Network for clinical trials has improved the quantity and quality of large multicenter clinical trials for ALI/ARDS patients. New research is continuing and tools such as genetic analysis, genomics and proteomics may offer more value for patients in the future.

\section{References}

Abraham E. Neutrophils and acute lung injury. Cr Care Med 2003 Apr; 31(4 Suppl):S 195-9

Ashbaugh DG, Bigelow DB, Petty TL \& Levine BE. Acute respiratory distress in adults. The Lancet, Sat 12 August 1967 Crit Care Resusc 2005 Mar;7(1):60-1

Bauer TT, Monton C, Torres A, Cabello H, Fellela X, Maldonado A, Nicolas JM \& ZavalaE. Comparison of systemic cytokine levels in patients with acute respiratory distress syndrome, severe pneumonia and controls. Thorax 2000 Jan; 55(1):46-52

Bdeir, K, Higazi AA, Kulikovskaya I, Christofidou-Solomidou M, Vinogradov SA, Allen TC, Idell S Linzmeier R, Ganz T \& Cines DB. Neutrophil a-Defensins Cause Lung Injury by Disrupting the Capillary Epithelial Barrier. AJRCCM Am J Respir Crit Care Med 2010 May;181(9): 935- 46

Bernard GR, Artigas A, Brigham KL, Carlet J, Falke D, Hudson L, Lamy M, LeGall JR, Morris A \& Spragg R. Report of the American-European Consensus conference on acute respiratory distress syndrome: definitions, mechanisms, relevant outcomes, and clinical trial coordination. Consensus Committee. J Crit Care 1994 Mar;9(1):72-81

Blackwell TS, Blackwell TR, Holden EP, Christman BW \& Christman JW. In vivo antioxidant treatment suppresses nuclear factor-kappa B activation and neutrophilic lung inflammation. J Immuno. 1996 Aug 15;157(4):1630-7

Braun, C, Hamacher J, Morel DR, Wendel A \& Lucas R. Dichotomal Role of TNF in Experimental Pulmonary Edema Reabsorption. J Immunol 2005 Sep; 175(5):3402-8

Calfee CS, Ware LB, Glidden DV, Eisner MD, Parsons PE, Thompson BT \& Matthay MA, National Heart, Blood, and Lung Institute Acute Respiratory Distress Syndrome Network. Use of risk reclassification with multiple biomarkers improves mortality prediction in acute lung injury. Crit Care Med 2011 Apr;39(4):711-7

Costello ML, Mathieu-Costello O \& West JB. Stress failure of alveolar epithelial cells studied by scanning electron microscopy. Am Rev Respir Dis 1992 Jun;145(6):1446-55

Downey GP, Kong Q, Kruger J, Dedhar S \& Cherapanov V. Regulation of Neutrophil Activation in Acute Lung Injury. Chest 1999 Jul;116(1suppl):46S-54S

Elia N, Tapponnier M, Matthay MA, Hamacher J, Pache JC, Brundler MA, Totsch M, DeBaetselier PD, Fransen L, Fukuda N, Morel DR \& Lucas R. Functional identification of the alveolar edema reabsorption activity of murine tumor necrosis factor-alpha. Am J Respir Crit Care Med 2003 Nov;168(9):1043-50 
Fein A, Grossman RF, Jones JG, Overland E, Pitts L, Murray JF \& Staub NC. The value of edema fluid protein measurement in patients with pulmonary edema. Am J Med 1979 Jul;67(1):32-8

Folkesson HG \& Matthay MA. Alveolar Epithelial Ion and Fluid Transport Recent Progess. Am J Respir Cell Mol Biol 2006 Jul;35(1):10-19Fremont RD, Kallet RH, Matthay MA \& Ware LB. Postobstructive Pulmonary Edema: A case for Hydrostatic Mechanisms. Chest 2007 Jun;131;1742-46

Fukuda N, Jayr C, Lazrak A, Wang Y, Lucas R, Matalon S \& Matthay MA. Mechanisms of TNF-a stimulation of amiloride sensitive sodium transport across alveolar epithelium. Am J Physiol Lung Cell Mol Physiol 2001 Jun; 280(6): L1258-65

Gantor CC, Jakob SM \& Takala J. Pulmonary capillary pressure. Minerva Anestesiol 2006 Janfeb;72(1-2):21-36

Geerts, Jorens, Willems, DeLey \& Slegers. Natural inhibitors of neutrophil function in acute respiratory distress syndrome. Crit Care Med 2001 Oct;29(10):1920-24

Grommes J \& Soehnlein O. Contribution of Neutrophils to Acute Lung Injury. Mol Med 2011 Mar;17(3-4)293-307

Hamacher J, Stammberger U, Roux J, Kumar S, Yang G, Xiong C, Schmid RA, Fakin RM, Chakraborty T, Hossain HM, Pittet JF, Wendel A, Black SM \& Lucas R. The lectin like domain of tumor necrosis factor improves lung function after rat lung transplantation Potential role for a reduction in reactive oxygen species generation Crit Care Med 2010 Mar 38(3):871-8

Hasko G, Xu DZ, Lu Q, Nemeth, ZH, Jabush J, Berezina TL, Zaets SB, Csoka B \& Deitch EA. Adenosine A2A receptor activation reduces lung injury in trauma/hemorrhagic shock. Crit Care Med. 2006 Apr;34(4): 1119-25

Holter JF, Weiland JE, Pacht ER, Gadek JE \& Davis WB. Protein Permeability in the Adult Respiratory Distress Syndrome Loss of Size Selectivity of the Alveolar Epithelium. J Clin Invest 1986 Dec; 78(6): 1513-22

Hyers TM, Tricomi SM, Dettenmeier PA \& Fowler AA. Tumor necrosis factor levels in serum and bronchoalveolar lavage fluid of patients with the adult respiratory distress syndrome. Am Rev Respir Dis 1991 Aug;144(2):268-71

Kaner RJ, Ladetto JV, Singh R, Fukuda N \& Matthay MA, Crystal RG. Lung over-expression of the Vascular Endothelial Growth Factor Gene Induces Pulmonary Edema. Am J Respir Cell Mol Biol 2000 Jun; 22(6):657-664

Kapoor MC. Negative pressure pulmonary oedema. Indian J Anaesth. 2011 Jan; 55(1):10-1

Lucas R, Verin AD, Black SM \& Catravas JD. Regulators of endothelial and epithelial barrier integrity and function in acute lung injury. Biochem Pharmacol2009 Jun 15;77(12): 1763-72

Maggiorini. Prevention and Treatment of High Altitude Pulmonary Edema. Prog CardiovascDis 2010 May-Jun;52(6):500-6Marino P. The ICU Book third edition. Lippincott Williams and Wilkins 2007 ISBN-13: 978-0-7817-4802-5

Marinovic, Ljubkovic, Obad, Breskovic, Salamunic, Denoble \& Dujic. Assessment of Extravascular Lung Water and Cardiac Function in Trimix SCUBA Diving. Med SciSports Exerc 2010 Jun;42(6):1054-61

Nakamura T, Sato E, Fujiwara N, Kawagoe Y, Maeda S \& Yamagishi S. Increased levels of soluble receptor for advanced glycation end products (sRAGE) and high mobility 
group box 1 (HMGB1) are associated with death in patients with acute respiratory distress syndrome. Clin Biochem2011 Jun;44(8-9): 601-4

Ono N, Mizuno R \& Ohhashi T. Effective permeability of hydrophilic substances through walls of lymph vessels: roles of endothelial barrier. Am J Physiol Heart Circ Physiol 2005 Oct;289(4): H1676-82

Pathak V, Rendon IS \& Ciubotaru RL. Recurrent Negative Pressure Pulmonary Edema. Clin MedRes 2011 Jun;9(2):88-91Petrache I, Birukova A, Ramirez SI, Garcia JG \& Verin AD. The Role of the Microtubules in Tumor Necrosis Factor-a-Induced Endothelial Cell Permeability. Am J Respir Cell Mol Biol 2003 May;28(5):574-81

Pons, Blickenstorfer, Oechslin, Hold, Greminger, Franzeck \& Russi. Pulmonary edema in healthy persons during scuba-Diving and swimming, Eur Respir J, 1995 May;8(5):762-7

Sartori C, Rimoldi SF \& Scherrer U. Lung fluid movements in hypoxia. Prog Cardiovasc Dis. 2010 May;52(6):493-9

Schutte H, Lohmeyer, J, Rosseau S, Ziegler S, Siebert C, Kielisch H, Pralle H, Grimminger F, Morr H \& Seeger W. Bronchoalveolar and systemic cytokine profiles in patients with ARDS, severe pneumonia and cardiogenic pulmonary edema. Eur Respir J 1996 Sep; 9(9):1858-67

Schwartz, Maroo, Malhotra \& Kesselman. Negative Pressure Pulmonary Hemorrhage. Chest 1999; Apr;115(4):1194-97

Slade JB, Hattori T, Ray CS, Bove AA \& Cianci P. Pulmonary Edema Associated with Scuba Diving: Case Reports and Review. Chest 2001Nov;120(5);1686-94

Slutsky AS, Tremblay LN. Multiple System Organ Failure Is Mechanical Ventilation a Contributing Factor? Am J Respir Crit Care Med 1998 Jun;157(6 Pt 1):1721-5

Staub NC, Hitoshi N \& Pearce ML. Pulmonary edema in dogs, especially the sequence of fluid accumulation in lungs. J Appl Physiol 1967 22(2):227-240

Steinberg KP, Milberg JA, Martin TR,Maunder RJ, Cockrill BA \& Hudson LD. Evolution of bronchoalveolar cell populations in the adult respiratory distress syndrome. Am J Respir Crit Care Med 1994 Jul;150(1):113-22

Swenson, Miggiorini, Mongovin, Gibbs, Greve, Mairbaurl \& Bartsch. Pathogenesis of High Altitude Pulmonary Edema-Inflammation is not an etiologic Factor. JAMA, 2002 May; 287(17):2228-35

The Acute Respiratory Distress Syndrome Network. Ventilation with lower tidal volumes as compared with traditional tidal volumes for acute lung injury and the acute respiratory distress syndrome. N Engl J Med 2000 May;342(18):1301-8

Tzouvelekis A, Pneumatikos I \& Bouros D. Serum biomarkers in Acute Respiratory Distress Syndrome: an ailing prognosticator. Respir Res 2005 Jun;6:62

Umapathy NS, Fan ZH, Zemskov EA, Alieva IB, Black SM \& Verin AD. Molecular mechanisms involved in adenosine induced endothelial cell barrier enhancement. Vascul Pharmacol2010 May; 52(5-6):199-206

Ward PA. Role of Complement, Chemokines, and Regulatory Cytokines in Acute Lung Injury. Ann NY Acad Sci. 1996 Oct 31;796:104-12

Ware LB, Fremont RD, Bastarache JA, Calfee CS \& Matthay MA. Determining the aetiology of pulmonary oedema by the oedema fluid-to-plasma protein ratio. Eur Respir J 2010 Feb;35(2): 331-7 
Weiland JE, Davis WB, Holter JF, Mohammed JR, Dorinsky PM \& Gadek JE. Lung neutrophils in the adult respiratory distress syndrome. Am Rev Respir Dis 1986 Feb;133(2):218-5

Wolfson RK, Chiang ET \& Garcia JG. HMGB1 induces human lung endothelial cell cytoskeletal rearrangement and barrier disruption. Microvas Res 2011 Mar;81(2): 89-97

Ware LB \& Matthay MA. Clinical practice. Acute pulmonary edema. N Engl J Med. 2005 Dec; 353(26):2788-96

Zimmerman GA \& McIntyre TM. PAF, ceramide and pulmonary edema: alveolar flooding and a flood of questions. Trends Mol Med2004 Jun;10(6):245-8 


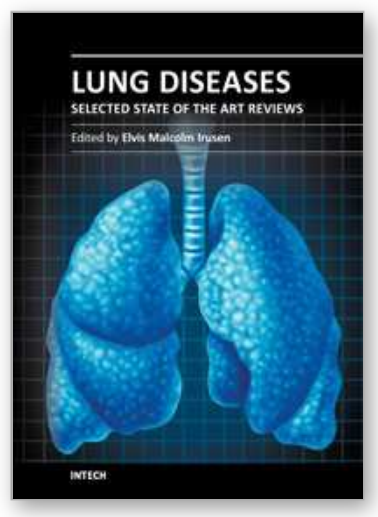

\section{Lung Diseases - Selected State of the Art Reviews}

Edited by Dr. Elvisegran Malcolm Irusen

ISBN 978-953-51-0180-2

Hard cover, 690 pages

Publisher InTech

Published online 02, March, 2012

Published in print edition March, 2012

The developments in molecular medicine are transforming respiratory medicine. Leading clinicians and scientists in the world have brought their knowledge and experience in their contributions to this book. Clinicians and researchers will learn about the most recent advances in a variety of lung diseases that will better enable them to understand respiratory disorders. This treatise presents state of the art essays on airways disease, neoplastic diseases, and pediatric respiratory conditions. Additionally, aspects of immune regulation, respiratory infections, acute lung injury/ARDS, pulmonary edema, functional evaluation in respiratory disorders, and a variety of other conditions are also discussed. The book will be invaluable to clinicians who keep up with the current concepts, improve their diagnostic skills, and understand potential new therapeutic applications in lung diseases, while scientists can contemplate a plethora of new research avenues for exploration.

\section{How to reference}

In order to correctly reference this scholarly work, feel free to copy and paste the following:

J. Gonzales and A. Verin (2012). Non-Cardiogenic Pulmonary Edema, Lung Diseases - Selected State of the Art Reviews, Dr. Elvisegran Malcolm Irusen (Ed.), ISBN: 978-953-51-0180-2, InTech, Available from: http://www.intechopen.com/books/lung-diseases-selected-state-of-the-art-reviews/non-cardiogenic-pulmonaryedema

\section{INTECH}

open science | open minds

\section{InTech Europe}

University Campus STeP Ri

Slavka Krautzeka 83/A

51000 Rijeka, Croatia

Phone: +385 (51) 770447

Fax: +385 (51) 686166

www.intechopen.com

\section{InTech China}

Unit 405, Office Block, Hotel Equatorial Shanghai

No.65, Yan An Road (West), Shanghai, 200040, China 中国上海市延安西路65号上海国际贵都大饭店办公楼 405 单元

Phone: +86-21-62489820

Fax: $+86-21-62489821$ 
(C) 2012 The Author(s). Licensee IntechOpen. This is an open access article distributed under the terms of the Creative Commons Attribution 3.0 License, which permits unrestricted use, distribution, and reproduction in any medium, provided the original work is properly cited. 